\title{
Wirtschaftliche Entwicklung und ökologische Belastbarkeit im Berggebiet (MAB Schweiz)
}

Mitte April 1978 wurde das Nationale Forschungsprogramm "Sozio-ökonomische Entwicklung und ökologische Belastbarkeit im Berggebiet (MAB Schweiz)" ausgeschrieben. Im folgenden Beitrag werden die forschungspolitische Ausgangslage, die wichtigsten Zielsetzungen und das Konzept zur Durchführung des Forschungsprogrammes näher erläutert.

\section{MAB: EINE NATIONALE AUFGABE IN EINEM INTER-} NATIONALEN RAHMEN

$M A B$ ist ein integrales und interdisziplinäres Forschungsprogramm der UNESCO, das mit der Umschreibung "Mensch und Biosphäre" (Man and Biosphere) uns die für die Zukunft entscheidenden Fragen der Tragfähigkeit und Belastbarkeit eines Raumes, des Gleichgewichtes zwischen $\mathrm{Na}$ turhaushalt und menschlicher Aktivität stellen will. 14 grosse Projekte, z.T. in geographischer Ordnung (tropische Regenwälder, Savannen, Wuisten, Steppen, Mittelmeergebiet, Tundren usw.), z.T. mit übergeordneter thematischer Orientierung (Städte, Küsten, Inseln, Gebirge usw.) tragen die gleiche Fragestellung in die reich differenzierten Teilräume unserer Erde. In 87 Staaten gibt es heute spezielle nationale Komitees, an ungefähr 400 Projekten wird gearbeitet. Diese Zahlen sollen in den nächsten zwei Jahren auf 100 nationale Institutionen mit über 500 Untersuchungsgebieten angehoben werden (UNESCO 1978: 20 und 155).

In diesem allzu weiten Rahmen hat sich die Schweiz, im Unterschied zu unseren Nachbarländern, entschlossen, nur in einem Projekt mitzuarbeiten. Das Gebirgsprogramm ( $\mathrm{Nr}$. 6: Man's impact on mountain ecosystems) zielt ganz genau auf eine unserer grossen nationalen Aufgaben hin: Das Suchen und Festlegen von Gleichgewichten zwischen der wirtschaftlich - gesellschaftlichen Entwicklung und der Tragfähigkeit unseres Lebensraumes.

\section{PROBLEME UND FUNKTION UNSERER BERGGEBIETE}

Sie lassen sich vereinfacht in drei Kategorien zusammenfassen

1. Schutz: Schutzfunktion im Sinne des ökologischen Landschaftsschutzes zur Erhaltung der Arten und Formenvielfalt der Natur- und Kulturlandschaft; aber auch Schutz vor Naturge- fahren, die in Form von Lawinen, Steinschlag, Rutschungen, Ueberschwemmungen usw. die touristische Erschliessung und das Kulturland der Täler und des Alpenvorlandes bedrohen.

2. Produktion: Die Berggebiete als land- und forstwirtschaftlicher Produktionsraum sind auch im volkswirtschaftlichen Rahmen wichtig. Zudem sind die Gebirgsregionen für die Wasserund Energieversorgung eines weiteren Umlandes von höchster Bedeutung.

3. Erholung: Die Vielfalt der Landschaft und des Klimas, die Nähe zu den grossen Bevölkerungszentren und die Attraktivität der Lebensund Wirtschaftsweise der Bergbevölkerung machen das Berggebiet zu einem der wichtigsten Erholungsräume unseres Landes und unserer Nachbarländer.

Durch die wirtschaftliche und technische Entwicklung in der Nachkriegszeit wurde unser Gebirgsraum immer mehr zum Konfliktgebiet in der Auseinandersetzung zwischen der Erhaltung naturnaher Areale, einer aktiven landwirtschaftlichen Bevölkerung und einer zunehmenden touristischen und technischen Erschliessung. Expandierender Tourismus und technische Eingriffe beträchtlichen Ausmasses (z.B. der Bau von Hochleistungsstrassen, Speicherbecken usw.) haben hicht nur die traditionelle Landnutzung verändert, sondern zugleich tiefgreifende Umstrukturierungen im sozialen, wirtschaftlichen und kulturellen Bereich herbeigeführt. Diese Veränderungen im alpinen Raum sind sehr eindrücklich am Beispiel von Obergurgl dargestellt worden, indem das geschlossene, autarke System um 1860 dem offenen, komplexen System der Gegenwart gegenübergestellt wird (siehe Fig. 1).

Der mit dieser Entwicklung verbundene Funktionswandel der Berglandschaft hat eingespielte Gleichgewichte zwischen dem Naturhaushalt und der menschlichen Nutzungs- und Kulturtätigkeit gestört und verschoben. Die heutigen, völlig veränderten Einwirkungen auf die verschiedenen Naturund Landschaftsbereiche sind in ihren direkten Wirkungen offensichtlich, in den möglichen mittelund langfristigen Folgewirkungen aber kaum abschätzbar.

Prof. Dr. B. Messerli und Dr. P. Messerli, Geographisches Institut der Universität Bern, Hallerstrasse 12, 3012 Bern. 


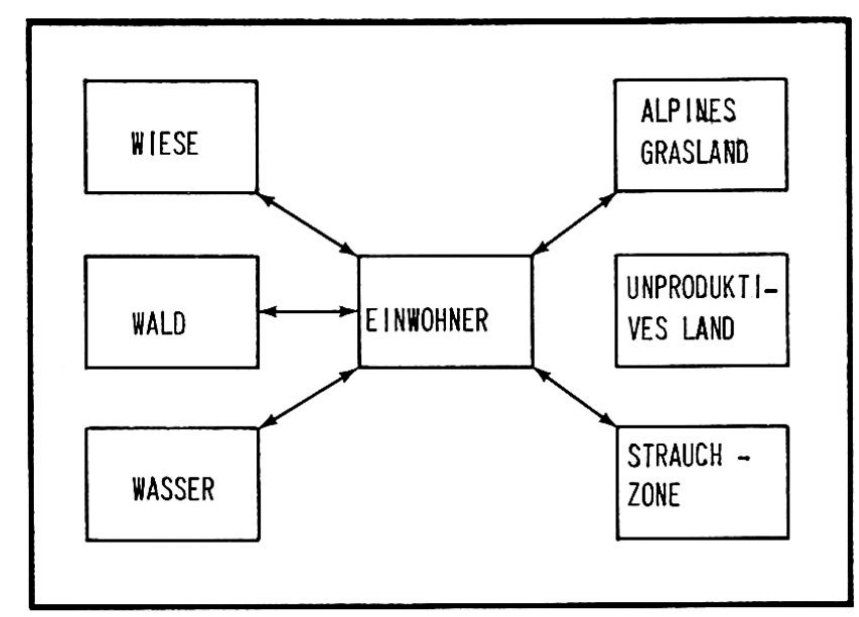

OBERGURGL 1860: ge s c h l o s s e n e s System
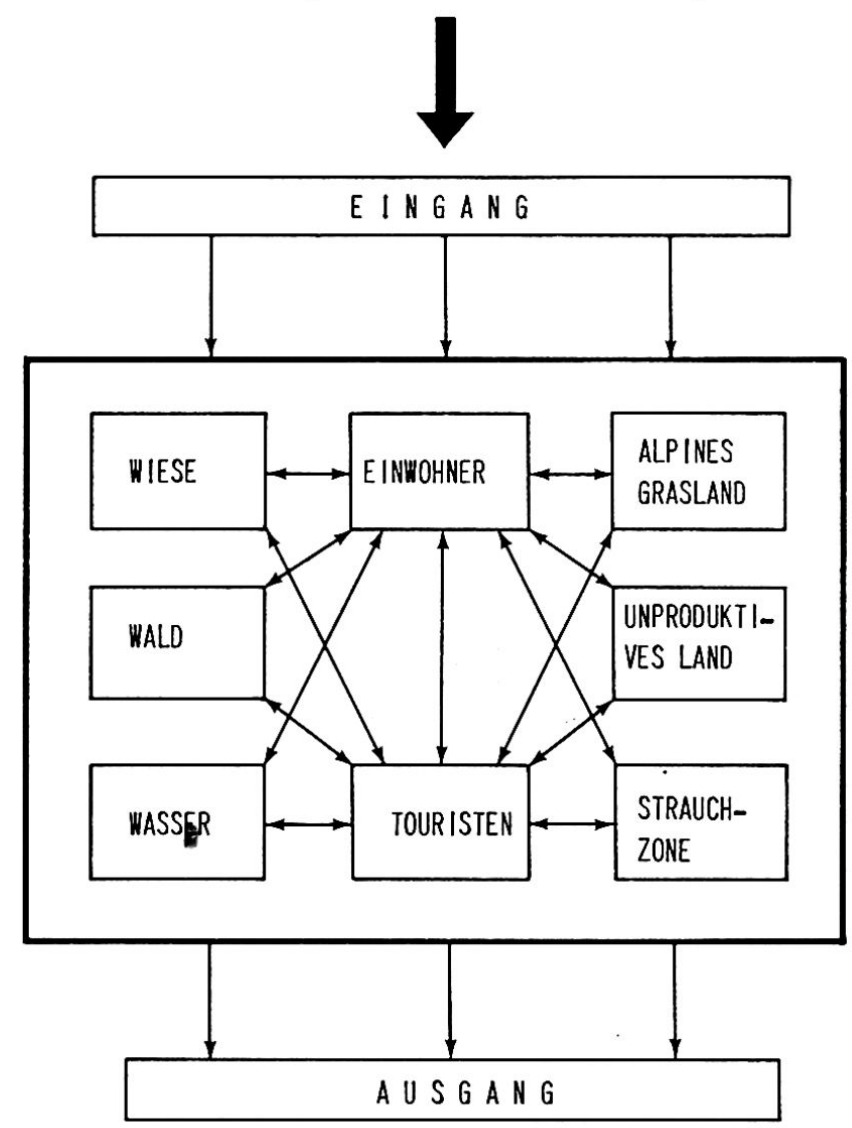

OBERGURGL heute: of $f$ e $n$ e s System

Fig. 1: Die Entwicklung vom yeschlosscnen zum offenen System (Bsp. Obergurgl, Tirol). (aus MOSER, W., 1975)

Wenn unser Alpenraum auch den nachfolgenden Generationen der Bergbevölkerung als Lebensraum und weiten Bevölkerungskreisen als Erholungsraum dienen soll, dann mussen die Nutzungsansprüche auf die Leistungsfähigkeit des Naturhaushaltes abgestimmt werden, d.h. die sozioökonomische Entwicklung muss in ein regional differenziertes Gleichgewicht zur ökologischen Belastbarkeit gebracht werden. Daraus ist eine politische Zielsetzung abzuleiten, in der ein wesentlicher Auftrag an die Wissenschaft enthalten ist:
3. "LANGFRISTIGE SICHERUNG UNSERER BERGGEBIETE ALS LEBENS-, WIRTSCHAFTS - UND ERHOLUNGSRAUM"

Ein Lebensraum, der nicht auch Wirtschaftsraum ist, ist tot. Ein Erholungsraum, der nicht auch Lebens- und Wirtschaftsraum ist, ist steril. Ein Wirtschaftsraum, der nicht auch Lebensraum ist, gefährdet die Umwelt und die Ressourcen.

Diese komplementären Funktionen müssen in ein dem Naturraum angepasstes Verhältnis gebracht werden, so dass

- das natürliche Produktionspotential in Form der verschiedenen Ressourcen (landwirtschaftliche Produktionsfläche, Wald, Wasser usw.) langfristig gesichert werden kann

- eine aktive Bevölkerung erhalten werden kann, die bereit und in der Lage ist, diese Ressourcen zu nutzen.

- eine angemessene Umweltqualität, verstanden als natürliches, soziales und kulturelles Milieu, dieser Bevölkerung und den Erholungssuchenden langfristig erhalten bleibt.

Zur Wahrnehmung der konkreten politischen Aufgaben im Sinne der Entwicklungsplanung und -steuerung liegen heute sektorielle Grundlagen vor. Diese sind aber zu wenig abgestützt auf ein vertieftes Verständnis der komplexen Beziehungen zwischen dem Naturhaushalt einerseits und der sozio-ökonomischen Entwicklung andererseits.

Im Rahmen des Nationalen Forschungsprogrammes "Sozio-ökonomische Entwicklung und ökologische Belastbarkeit im Berggebiet" sind deshalb Grundlagen bereitzustellen, die praktische Entscheidungshilfen für eine die Belastungsgrenzen nicht überschreitende, also nachhaltige Nutzung unserer Berggebiete abgeben können. Aus dieser Forderung sind die folgenden wissenschaftlichen Zielsetzungen abzuleiten:

1. Funktionsweise eines regionalen ökonomischökologischen Systems

Die Entwicklung und Erschliessung einer alpinen Region ist ein Prozess, der durch Impulse von aussen und von innen ausgelöst und bestimmt wird. Dieser Prozess, der zudem eigendynamischen Gesetzmässigkeiten folgt, ist durch typische Ereignisfolgen zu charakterisieren und auf die entscheidenden Grössen hin zu analysieren (Impulsgrössen, Regelgrössen, kritische Grössen usw.).

2. Belastbarkeit eines regionalen ökonomischökologischen Systems

Prozesse und Beziehungen sind besonders auf Grenzbedingungen hin zu untersuchen, bei denen irreversible Systemveränderungen eintreten können (z.B. Verlust der Regenerationsfähigkeit des Bodens und der Pflanzendecke, Fernbleiben der Touristen, Abwanderung der Bevölkerung, Zusammenbruch der Berglandwirtschaft usw.). Davon ausgehend ist abzuklären, ob Belastungsgrenzen im ökologischen und im sozio-ökonomischen Bereich definiert werden können. 
3. Steuerbarkeit eines regionalen ökonomischökologischen Systems

Aus den exemplarischen Erkenntnissen über Funktionsweise und Belastbarkeit des Systems sind Entscheidungsgrundlagen für eine optimale Nutzung der natürlichen Ressourcen unserer Berggebiete zu erarbeiten.

Die Optimierung erfolgt unter Berücksichtigung der verschiedenen Belastungsgrenzen und im Hinblick auf die Ansprüche und Entwicklungsvorstellungen der betroffenen Bevölkerung und der Touristen.

\section{METHODISCHE PROBLEME EINER INTEGRALEN BE- TRACHTUNG}

Mit der Erschliessung der Berggebiete setzte in vielen Fällen eine Entwicklung ein, wie sie in Fig. I symbolhaft dargestellt ist: Ein weitgehend autarkes, geschlossenes System wandelt sich in ein offenes, durch Fremdeinflüsse bestimmtes System. Dieses ist gekennzeichnet durch Ein- und Ausgänge von Personen (Touristen, Pendler etc.), Guitern, Kapital, Information und durch eine zunehmende Komplexität und Vernetzung aller Elemente. Aus einem überblickbaren Lebensraum mit angepassten Funktionen wird ein Raum mit unüberblickbaren Funktionsverflechtungen, die kaum mehr auf die gegebenen natürlichen, gesellschaftlichen und wirtschaftlichen Strukturen ausgerichtet sind und zu Konflikten und Belastungen führen. Korrigierende
Eingriffe, obschon lokal von einiger Wirkung, verschieben aber oft Belastungen nur an eine andere Stelle des Systems, weil sie sich über schwer erkennbare Wirkungsketten mit verschiedenen Zeitkonstanten auswirken.

Problemanalysen und Läsungsvorschläge müssen deshalb auf den ganzheitlichen Zusammenhängen eines Mensch - Umweltsystems im Berggebiet basieren, wie es in Fig. 2 vereinfacht und schematisch dargestellt ist. Funktionsweise, Belastbarkeit und Steuerbarkeit eines solchen räumlich-funktionalen Systems stellen grundsätzliche methodische Probleme, auf die wir im folgenden kurz eintreten:

\subsection{Systemabgrenzung (inhaltlich, räumlich, zeitlich)}

Die inhaltliche Bestimmung eines Systems hängt von den vorgegebenen Fragestellungen $a b$. Da in unserem Fall die Wechselbeziehungen zwischen dem ökologischen und dem sozio-ökonomischen Bereich im Vordergrund stehen, sind durch diese beiden Teilsysteme die wesentlichen Grössen definiert. Für die Steuerbarkeit des Gesamtsystems sind neben internen auch externe Einflussgrössen zu berücksichtigen. Da zudem eine möglichst genaue Erfassung der Beziehungen auch innerhalb der Teilsysteme angestrebt wird, sind diese möglichst detailliert zu beschreiben.

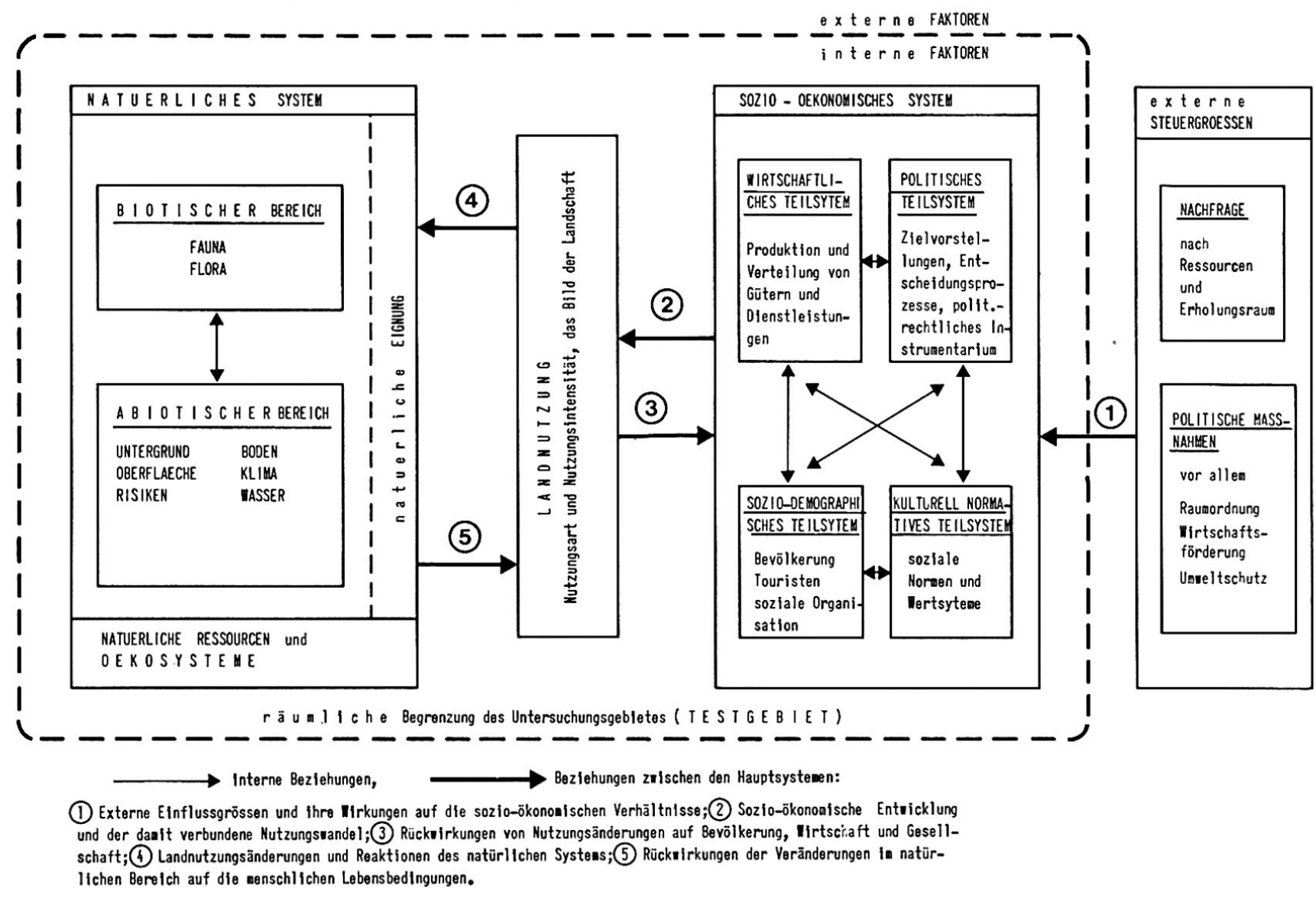

Fig. 2: Schematische Darstellung eines regionalen ökonomisch-ökologischen Systems 
Bei der räumlichen Abgrenzung müssen die Wirtschaftsflächen und der Erholungsraum einer oder mehrerer Siedlungen mit den charakteristischen Höhenstufen berücksichtigt werden. Die Abgrenzung hat deshalb nach natur- und wirtschaftsräumlichen Kriterien zu erfolgen.

Jedes räumliche System hat eine zeitliche Dimension, man spricht von gewachsener Siedlung und Landschaft. Diese Dimension ist nicht nur zum Verständnis der heutigen Nutzungssituation notwendig, sie deckt auch Steuermechanismen und Entscheidungsprozesse auf, die in der Vergangenheit die räumliche Entwicklung bestimmt haben. Der zeitliche Rahmen der Untersuchung muss deshalb der Geschichte des betrachteten Raumes und seiner Bewohner angepasst sein und die gesellschaftlichen, rechtlichen und nutzungsmässigen Bedingungen des heutigen Zustandes aufzeigen.

\subsection{Beziehungsanalyse und Systemvernetzung} (Funktionsweise des Systems)

Diese beiden Schritte sind methodisch anspruchsvoll, und bilden den zentralen Teil des Programms.

Die in Fig. 2 dargestellten Hauptbeziehungen lassen sich in der folgenden stark vereinfachten Wirkungskette zusammenfassen: Externe und interne Entwicklungsimpulse übertragen sich über das sozio-ökonomische System auf die Landnutzung und den Naturhaushalt. Die dadurch ausgelösten Veränderungen können sich sowohl direkt (Sicherheit, Gesundheit, Wohlbefinden usw.) als auch indirekt (über die Produktionsbedingungen, Nutzungsbeschränkungen usw.) auf den Menschen auswirken, der dadurch zur Anpassung seiner Nutzungsstrategien veranlasst wird. Diese Wirkungskette mit ganz unterschiedlichen Zeitkonstanten innerhalb und zwischen den Teilsystemen resultiert aus der Vernetzung der Systemelemente. Die modellmässige Erfassung dieser Zusammenhänge ist eine wesentliche Aufgabe dieses Programmes. Sie wird grundsätzlich erschwert durch die Tatsache, dass die Natur selbst (Naturgefahren, Katastrophen) und vor allem der Mensch mit seinen Entscheiden auf politischer, wirtschaftlicher und gesellschaftlicher Ebene immer wieder in Form von "Zufallsimpulsen" im Systemablauf interveniert und damit eine regelhafte Darstellung nur in Grenzen möglich sein wird.

Im vorgegebenen Zeitraum von 5 Jahren können natürlich nicht sämtliche Beziehungen wissenschaftlich untersucht werden. Es ist aber zu bedenken, dass viele Zusammenhänge bereits untersucht sind und nur noch in konkreten Fallstudien überprüft und eingebaut werden müssen. Das Schwergewicht der Untersuchungen soll deshalb auf weniger bekannte Zusammenhänge, vor allem zwischen Landnutzung, Naturhaushalt und ökologischer
Stabilität durch gezielte Untersuchungen gelegt werden.

Das eigentliche methodische Hauptproblem liegt schliesslich beim Zusammenbau verschiedener Teilmodelle zu einem Gesamtmodell, das in seiner "Funktionsweise" überblickbar bleiben und das Erkennen der Ursachen von unerwünschten Systemveränderungen gestatten soll.

4.3. Grenzwerte, kritische Werte und Schwellenwerte (Belastbarkeit)

Als Orientierungshilfen und Entscheidungsgrundlagen sind Grenz- und Schwellenwerte von entscheidender Bedeutung. Schwellenwerte markieren eine untere, Grenzwerte eine obere Schranke. Dazwischen können bestimmte Grössen variieren, ohne dass sich wesentliche Veränderungen im System ereignen. Zum Beispiel ist eine Gemeinde politisch, wirtschaftlich und gesellschaftlich nicht mehr funktionsfähig, wenn eine Mindestbevölkerungszahl unterschritten wird oder es müssen neue Organisationsformen gefunden werden, wenn ein oberer Grenzwert überschritten wird. Solche Werte können verständlicherweise nur aus dem Systemverständnis heraus, unter Berïcksichtigung regionaler Bedingungen, sinnvoll ermittelt werden. Kritische Werte sollen, mit Hilfe eines Indikatorsystems frühzeitig erkannt, auf Fehlentwicklungen hinweisen, so dass zweckmässige Massnahmen vor Erreichen oder Ueberschreiten eines Grenz- oder Schwellenwertes ergriffen werden können.

\subsection{Modell-Simulation (Steuerbarkeit)}

Das Wesen jeder Simulation liegt darin, Handlungsweisen auf verschiedene Konsequenzen hin überprüfen zu können, ohne das reale System damit zu belasten. Soweit nun eine modellmässige Darstellung der wesentlichen Beziehungen zwischen den Komponenten eines räumlichen Systems möglich ist, kann dieses Instrument dazu verwendet werden, Wirkungsanalysen planerischer und politischer Massnahmen durchzuführen und diese bezüglich Ziel- und Systemkonformität zu beurteilen. Weiter können konkrete Entwicklungsvorstellungen der verschiedenen betroffenen Bevölkerungsgruppen oder bestimmte Entwicklungsmodelle auf die räumlichen, wirtschaftlichen und gesellschaftlichen Auswirkungen und Veränderungen hin untersucht, als planerische Szenerien dargestellt und mittels vorgängig festgelegter Zielkriterien bewertet werden.

Dieses Verfahren gestattet somit, Entscheidungsgrundlagen zu erarbeiten, die sich auf eine integrale Betrachtung der durch die verschiedenen Disziplinen gelieferten Entscheidungselemente abstuitzen. Der Entscheidungsträger wird dadurch in die Lage versetzt, die Konsequenzen seines Handelns zu "erfahren" und die entscheidenden Steuer- 
grössen und Selbstregulierungsmechanismen des Systems zu erkennen.

Die Suche nach Beziehungen und Prozessabläufen in einem komplexen System setzt also die Arbeit auf zwei Ebenen, der empirischen und theoretischen, gleichzeitig voraus. Ohne Modellvorstellungen über die Funktionsweise eines regionalen ökonomisch-ökologischen Systems ist eine sinnvolle Strukturierung der Beziehungen und damit der Problemvorstellungen nicht möglich. Durch die empirisch festgestellten Sachverhalte wird diese Modellvorstellung bestätigt, korrigiert und erweitert, woraus sich neue und präzisere Fragestellungen ergeben können. Das Modell als verbale, graphische oder mathematische Beschreibung des Systems wird somit zum zentralen Führungs- und Kontrol1instrument dieses Forschungsprogramms.

\section{AUSGEWAEHLTE TESTGEBIETE FUER EINE INTEGRALE BEARBEITUNG}

Die Realisierung eines integralen Forschungsansatzes verlangt einen konkreten räumlichen Bezugsrahmen, in dem die verschiedenen Systemvariablen eine genügende räumliche und zeitliche Informationsdichte aufweisen. Eine umfassende Datenbasis zur Charakterisierung der raumzeitlichen Variabilität der ökologischen und sozio-ökonomischen Grössen ist die wesentliche Voraussetzung zum Aufzeigen von Entwicklungsabläufen, zum Aufdecken von Wechselwirkungen und zur Ueberprüfung hypothetischer Sys temzusammenhänge.

Mit der Festlegung von 4 Testgebieten als verbindliche Untersuchungseinheiten soll die wissenschaftliche Bearbeitung von allem Anfang an räumlich fixiert werden. Dieses Vorgehen betont die Priorität der Untersuchungen und modellmässigen Darstellung des Gesamtsystems vor der Bearbeitung einzelner Problemstellungen. Bei der Auswahl der Untersuchungsgebiete musste der Forderung Rechnung getragen werden, dass der schweizerische Alpenraum zwischen 0st- und Westalpen sowohl regional als auch in bezug auf verschiedene touristische Entwicklungsmodelle möglichst repräsentativ vertreten ist. Dadurch sollen Vergleiche mit den Testgebieten der übrigen Alpenländer gewährleistet werden. Neben der spezifischen Eignung als alpines MAB-Testgebiet standen somit als wichtigste Kriterien die räumliche Verteilung, der Grad der bisherigen wissenschaftlichen Bearbeitung und die Erreichbarkeit von verschiedenen schweizerischen Hochschulzentren aus im Vordergrund. Nachfolgend wird die Forschungsthematik der 4 Testgebiet kurz charakterisiert.

\section{GRINDELWALD (Gemeinde Grindelwald, 170km2)}

Im traditionellen Fremdenverkehrsort mit einer einzigartigen Naturszenerie führte der in der zweiten Hälfte des letzten Jahrhunderts einsetzende Pioniertourismus sehr früh zur Oeffnung eines vorher geschlossenen Systems. Die Bergschaftsorganisationen, die bis in die Gegenwart ihren politischen Einfluss bewahren konnten, haben durch das Festhalten an altüberlieferten Nutzungsordnungen eine unkontrollierte touristische Erschliessung der Vorsassen und Alpgebiete weitgehend verhindert und der Berglandwirtschaft eine anerkannte Position erhalten.

Zu einer Konkurrenzierung der landwirtschaftlichen Produktionsfläche kam es allerdings im Bereiche der Dorf- und traditionellen Streusiedlungen durch eine starke Expansion des Zweitwohnungsbaus und der touristischen Infrastruktur. Diese zunehmenden räumlichen und wirtschaftlichen Verflechtungen zwischen Berglandwirtschaft und Tourismus kann zu wirtschaftlichen und gesellschaftlichen Ungleichgewichten und Nutzungskonflikten führen.

Am Beispiel Grindelwald soll deshalb das Wirkungsgefüge Berglandwirtschaft - Tourismus Naturraum untersucht werden, um Erkenntnisse über Stabilität und Belastbarkeit des natürlichen Systems und seine optimale Nutzung bei gleichzeitiger Erhaltung eines wirtschaftlichen und sozialen Gleichgewichtes zu gewinnen.

\section{ALETSCH (Aletschregion zwischen Massa und Märjelen, 60km2)}

Im krassen Unterschied zu Grindelwald erfolgte für den Grossteil der Region die 0effnung erst in den 50er Jahren mit der Erschliessung durch Strasse oder Seilbahn. Explosionsartig hat der Tourismus ein weitgehend autarkes Landwirtschaftssystem abgelöst und sich zum dominanten Wirtschaftsfaktor entwickelt. Die Bevölkerung hat ihren Wohnsitz von den traditionellen Dorfsiedlungen in die touristisch erschlossenen Alpsiedlungen verlagert. Der Substanzverlust im primären Sektor führte zum Zusammenbruch der dreistufigen landwirtschaftlichen Wirtschaftsform und zur Auflassung ungünstig gelegener Flächen. Diese Entwicklung hat zu zahlreichen Konflikten geführt, insbesondere zwischen den Interessen und der Notwendigkeit einer wirtschaftlichen Entwicklung und den Bestrebungen zur Erhaltung einer naturnahen Gebirgslandschaft.

An diesem Beispiel sind deshalb die Auswirkungen eines dynamisch expandierenden Tourismus in einer naturnahen Gebirgslandschaft aufzuzeigen und Entscheidungsgrundlagen für eine der landschaftsökologischen, wirtschaftlichen und gesellschaftlichen Belastbarkeit angepassten Entwicklung bereitzustellen.

\section{DAVOS (Dischmatal und Parsenngebiet, 90km2)}

In der Landschaft Davos wurden die Nebentäler von der touristischen Erschliessung des Haupttales und der Umschichtung des Höhenkurortes Davos zum internationalen Ferienort relativ wenig berührt. Währenddem die Landwirtschaft des Haupttales ihre Produktion auf die Versorgung der Touristenstadt Davos ausgerichtet hat, sind die traditionellen Nutzungsformen im Dischmatal weitgehend erhalten geblieben. Auf engstem Raum sind 
daher unterschiedliche Wirtschaftssysteme ausgeprägt und von einer touristischen Nutzung unterschiedlicher Intensität überlagert.

An diesem Beispiel soll deshalb mit Schwergewicht die 0ekologie der subalpinen und alpinen Stufe in Beziehung zum Wandel der Berglandwirtschaft, der Forstwirtschaft und des Tourismus untersucht werden, wobei Parsenngebiet und Dischmatal mit ihren Unterschieden in Exposition, geologischem Untergrund, Nutzung und touristischer Belastung verglichen werden sollen.

PAYS D'ENHAUT (Gemeinden Château-d'0ex, Rougemont, Rossinières und das Gebiet von Les Mosses, 200km2)

Trotz eines starken Rückganges der Betriebszahlen nimmt die Landwirtschaft wirtschaftlich, politisch und gesellschaftlich eine wichtige Stellung ein. Die landwirtschaftlichen Produktionsbedingungen sind im Vergleich mit den anderen Testgebieten besonders günstig, bedingt durch die geringe Höhenlage und die günstigen Wirtschaftsflächen im Haupttal und im Alpgebiet. Mechanisierung und Rationalisierung haben zur Aufgabe von Grenzstandorten und zur Nutzungsextensivierung der Voralpen und des Bergwaldes gefuihrt. Sommer- und Wintertourismus haben sich vor allem in Form eines "Familientourismus" in den Zentren Château-d'Oex und Rougemont entwickelt, das Gebiet von Les Mosses wurde als Zweitwohnungs- und Skigebiet erschlossen. Nutzungskonflikte entstanden vorab auf den von der Landwirtschaft bevorzugten Flächen, denen sowohl zur Erhaltung einer attraktiven Landschaft für Naherholung und Ferientourismus wie auch zur wirtschaftlichen Nutzung der natürlichen Ressourcen eine zentrale Bedeutung zukommt. Die langfristige Sicherung der Landwirtschaft hängt aber ganz wesentlich von einer ausgewogenen Entwicklung auch der übrigen Wirtschaftszweige $a b$.

An diesem Beispiel sollen verschiedene Entwicklungsmodelle unter Berücksichtigung der Naturgrundlagen, der wirtschaftlich-demographischen Verhältnisse und der Zukunftsvorstellungen der Bevölkerung auf die ökologischen, wirtschaftlichen und gesellschaftlichen Konsequenzen hin überprüft werden.

Durch die Vorgabe thematischer Schwerpunkte soll, immer im Rahmen des integralen Forschungsansatzes, den spezifischen Möglichkeiten und Bedürnissen der Testgebiete Rechnung getragen werden. Ihre Bearbeitung erfordert die Bildung multidisziplinärer Arbeitsgruppen. Die Entwicklung gemeinsamer Konzepte und Arbeitsmethoden, die auf einer operationellen Stufe zur Diagnose und Prognose von Zustand und Entwicklung leicht auf andere Gebiete übertragen werden können, ist ein weiteres wesentliches Ziel dieses Programmes. Da die heute zwingend notwendigen integralen Forschungsansätze nur durch Zusammenarbeit verschiedener Disziplinen gemeistert werden können, stellt dieses Programm hohe Anforderungen an die oft isoliert arbeitenden Wissen- schaftsbereiche. Eine fachliche und methodische Abstimmung der Arbeiten innerhalb und zwischen den Testgebieten und die Entwicklung neuer Formen interdisziplinärer Zusammenarbeit ist deshalb notwendig. Diese kann aber nur dann fruchtbar werden, wenn sie von einem gemeinsamen Interesse an einem bestimmten Raum und einer gemeinsamen Verantwortung für die künftige Entwicklung unserer Berggebiete getragen wird.

\section{GLEICHGEWICHT UND BELASTBARKEIT IM ALPINEN} RAUM, PROBLEME FUER POLITIK UND WISSENSCHAFT

In drei Gedanken sollen die wesentlichen Probleme zusammengefasst werden:

Zum ersten sollte jedes Wachstum in genauer Kenntnis der Belastbarkeit vor sich gehen. Planer und Politiker müssen ihre Entscheide zumindest in der Zeitdimension der nächsten Generation sehen. Wollen wir unsere natürlichen Ressourcen auch dem nächsten Jahrhundert erhalten, brauchen wir mit hoher Dringlichkeit ein ökologisches Management der wirtschaftlichen Entwicklung.

Zum zweiten sollten wir daran denken, Optionen für die Zukunft offen zu lassen. Irreversible Prozesse und irreversible Schäden müssen minimalisiert werden. Wissenschaft, Technologie und wirtschaftliche Entwicklung enthalten oder sind kumulative Prozesse, die nicht auf Kosten der Landschaft und der verfügbaren Bodenflächen ablaufen dürfen. Verfügbarer Boden ist die einzige Ressource, deren Grösse strikt und definitiv limitiert ist, national und global gesehen.

Zum dritten ist die Nutzung und Uebernutzung des Kapitals "Landschaft und Natur" nicht nur eine Funktion der wirtschaftlichen Aktivität im allgemeinen, sondern ihrer Formen, ihrer räumlichen Verteilung und ihrer Technologien im besonderen. Daraus müssen politische Konsequenzen gezogen werden: Umwelt und Naturhaushalt sind nicht nur einige zusätzliche Faktoren in einer allgemein dominierenden wirtschaftlichen Denk- und Arbeitsweise. Diese Elemente sind vielmehr die Basis, auf die sich alle Planungsentscheide beziehen müssen. Schliesslich geht es auch um die Erhaltung von Werten, die vielleicht momentan nicht wirtschaftlich nutzbar sind. Dabei kann "Schützen" zur Wertsteigerung einer Landschaft beitragen und vor allem zur Offenhaltung von Optionen für die Zukunft von entscheidender Bedeutung sein.

All diese Postulate verlangen aber eine intensivere Zusammenarbeit zwischen Naturwissenschaften und Sozial- und Wirtschaftswissenschaften, eine engere Zusammenarbeit zwischen Wissenschaft und ortsansässiger Bevölkerung und letztlich eine bessere Zusammenarbeit zwischen Wissenschaft und politischen Entscheidungsträgern.

\section{ZUSAMMENARBEIT DER ALPENLAENDER UND INTERNA- TIONALE BEDEUTUNG}

Entwicklung und Erhaltung unseres alpinen Lebens- 
raumes sind für die Schweiz nicht nur eine nationale, sondern auch eine internationale Aufgabe. Alle Alpenländer haben im Rahmen des MAB-Programmes ihre Testgebiete bezeichnet und die Arbeit aufgenommen. Eine fachliche und methodische Koordination ist unerlässlich, auch wenn Unterschiede in der Dauer der laufenden Arbeiten und in den verfügbaren finanziellen Mitteln bestehen. Denn alle stehen vor den gleichen schwierigen und vielleicht allzu hoch gesteckten Zielfragen, wie man ökologische und ökonomische Daten in einen Zusammenhang bringt, wie man Unvergleichbares vergleichbar macht und wie man letztlich in einem komplexen, dynamischen System die Funktionsweise verstehbar, die Belastungen definierbar und die Steuerbarkeit "entscheidbar" macht.

Schliesslich könnten aus diesen Projekten Teilbeiträge an die Gebirgsprobleme der Dritten Welt herauswachsen, denn grundsätzlich sind alle Gebirge der Welt durch die vergleichbaren Grundelemente der klimatisch und reliefbedingten Empfindlichkeit der 0ekosysteme gekennzeichnet. Bedenken wir bloss, dass ca. 10\% der Weltbevölkerung in Gebirgen lebt, dass aber 40\% in irgendeiner Weise von Gebirgen abhängen (MUELLER-HOHENSTEIN, K., 1974: 17 - 21), dann sollte diese globale Bedeutung der Gebirge für das Gebirgsland Schweiz eine gewisse Verpflichtung sein.

Zusammengefasst aber liegt gerade in diesem stufenweisen Aufbau das Faszinierende dieser
Forschungsprogramme: Wir arbeiten im Rahmen eines internationalen Programmes an einer nationalen Aufgabe und wir hoffen, durch die Lösung nationaler Aufgaben einer internationalen Verpflichtung nachzukommen.

\section{LITERATURNACHWE IS}

MOSER, W., 1975: Einige Erfahrungen mit dem Tourismus in den Alpen - das Oekosystem Obergurgl. Schriftenreihe des Alpeninstituts München, H. 3

MUELLER-HOHENSTEIN, K., (Editor), 1974: International workshop on the development of mountain environment, Munich 8-12 Dec. 1974. German Foundation for International Development, Food and Agriculture Development Centre

NATIONALE FORSCHUNGSPROGRAMME, 1978: Ausführungsplan zum NFP "Sozio-ökonomische Entwicklung und ökologische Belastbarkeit im Berggebiet (MAB Schweiz)". Schweizerischer Nationalfonds zur Förderung der wissenschaftlichen Forschung, Abt. Nationale Programme, Bern

UNESCO, 1978: Chronique UNESCO No 1, Projet de Programme 20 C 5, Paris

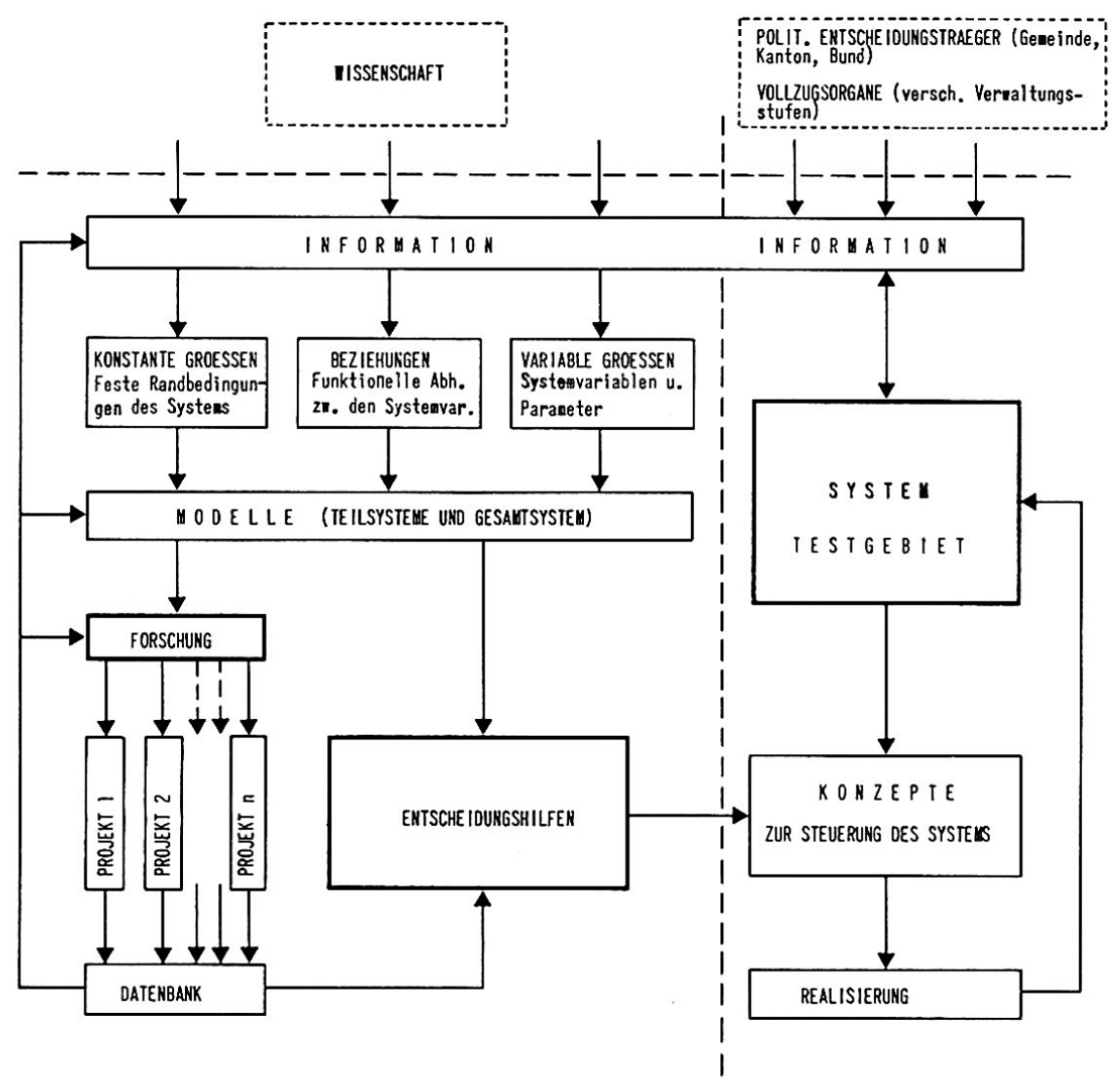

Fig. 3: Wissenschaft und Politik: Umsetzung der Wissenschaftlichen Erkenntnisse in planerische und politische Entscheide (abgeändert aus MOSER, W., 1975) 


\section{ZUSAMMENFASSUNG}

Das internationale UNESCO Programm. "Mensch und Biosphäre" (MAB) wird in seiner Zielsetzung und allgemeinen Bedeutung kurz umrissen. Der schweizerische Beitrag gilt dem Projekt Nr. 6: der Einfluss menschlicher Aktivitäten auf Gebirgsökosysteme im Rahmen des Nationalen Forschungsprogrammes "sozioökonomische Entwicklung und ökologische Belastbarkeit im Berggebiet". Mit einem integralen und interdisziplinären Forschungsansatz soll das Beziehungsgefüge zwischen Berglandwirtschaft, Tourismus und Naturraum an Fallbeispielen exemplarisch untersucht werden, um wichtige Mechanismen der räumlichen, wirtschaftlichen und gesellschaftlichen Entwicklung aufzudecken. Grenzen der Nutzung und Belastbarkeit eines räumlichen Systems sollen erkannt und damit Entscheidungsgrundlagen zur langfristigen Sicherung und Erhaltung unserer Berggebiete als Lebens-, Wirtschafts- und Erholungsraum erarbeitet werden. Die bei der Realisierung eines solch umfassenden Ansatzes auftretenden fachlichen und methodischen Probleme werden diskutiert und die Notwendigkeit hervorgehoben, neue Formen interdisziplinärer Zusammenarbeit zu entwickeln. Vier Testgebiete im schweizerischen Alpenraum (Aletsch, Davos, Grindelwald und Pays-d'Enhaut) geben dazu den konkreten räumlichen Bezugsrahmen. Sie sollen ermöglichen, die gewonnenen Erkenntnisse durch Vergleich mit den Verhältnissen in den MAB-6 Testgebieten der übrigen Alpenländer in ihrer räumlichen Bedeutung zu gewichten.

\section{RESUME}

Les buts et la signification générale du programme international de l'UNESCO intitulé "L'homme et la biosphère" (MAB) sont brièvement expliqués. La contribution suisse porte sur le projet No 6 - consacré à l'influence de 1 'homme et de ses activités sur les écosystèmes montagneux - dans le cadre du programme national de recherche "Développement socio-économique et charge écologique dans les régions de montagne". Grâce à une approche globale et interdisciplinaire, il est prëvu d'étudier l'ensemble des relations entre l'agriculture de montagne, le tourisme et les facteurs naturels sur la base de cas exemplaires; il s'agit de découvrir d'importants mécanismes du développement spatial, économique et social. On cherchera à établir les limites dans l'usage et la charge d'un système spatial donné, afin de fournir des critères de décision pour la conservation à long terme de nos régions de montagne, en tant qu'espaces de production, d'habitation et de délassement. Les problèmes de fond et de méthode qui surgissent lors de la réalisation d'un programme embrassant un domaine aussi vaste sont discutés; on souligne la nécessité de développer de nouvelles formes de coopération interdisciplinaire. Quatre airestests choisies dans les Alpes suisses (Aletsch, Davos, Grindelwald, Pays-d'Enhaut) fournissent le cadre de référence. Elles doivent permettre de pondérer, du point de vue de leur signification spatiale, les résultats de la recherche avec les conditions des aires-tests MAB- 6 dans les autres pays alpins.

\section{SUMMARY}

The international UNESCO - program MAB "Man and Biosphere" is briefly outlined with regard to its aims and general significance. The Swiss contribution centres on project no 6: "Impact of human activities on mountain ecosystems" within the framework of the national research program "Socioeconomic development and ecological capacity in mountain areas". With an integral and interdisciplinary approach, the relationship between mountain farming, tourism and nature has to be studied. Important mechanisms in urban, economic and social development should be exposed. The limits of land use and problems of carrying capacity of the environment have to be determined regarding to a long-term preservation of our mountain regions as living, economic and recreation area.

In the Swiss Alps, four test regions have been selected (Aletsch, Davos, Grindelwald, Paysd'Enhaut) to provide a concrete framework for field studies and to allow comparisons with the MAB- 6 test areas of the other alpine countries. 